\title{
Feasibility of Audio-Recording Consultations with Pregnant Australian Indigenous Women to Assess Use of Smoking Cessation Behaviour Change Techniques
}

\author{
Yael Bar-Zeev (D), ${ }^{1,2}$ Eliza Skelton, ${ }^{1}$ Michelle Bovill $\mathbb{D}^{1},{ }^{1}$ Maree Gruppetta ${ }^{\mathbb{D}}{ }^{1}$ \\ Billie Bonevski ${ }^{(D}{ }^{1}$ and Gillian S. Gould ${ }^{1}{ }^{1}$ \\ ${ }^{1}$ School of Medicine and Public Health, The University of Newcastle, Callaghan, New South Wales, Australia \\ ${ }^{2}$ Braun School of Public Health and Community Medicine, \\ The Hebrew University of Jerusalem-Hadassah Medical Organization, Israel
}

Correspondence should be addressed to Yael Bar-Zeev; yael.bar-zeev@mail.huji.ac.il

Received 17 September 2020; Revised 21 November 2020; Accepted 6 December 2020; Published 13 January 2021

Academic Editor: Michelle DiGiacomo

Copyright (C) 2021 Yael Bar-Zeev et al. This is an open access article distributed under the Creative Commons Attribution License, which permits unrestricted use, distribution, and reproduction in any medium, provided the original work is properly cited.

\begin{abstract}
Introduction. Behavioural counselling is an effective method to improve smoking cessation during pregnancy. Audio recordings of consultations have been used previously to assess fidelity in specialized smoking cessation services, but not in primary care. Aims. The study is aimed at assessing the feasibility of audio-recording smoking cessation counselling as part of an intervention in primary care settings and exploring the number and type of behaviour change techniques (BCTs) delivered. Methods. This study was a nested feasibility study within a larger trial. Health providers (HPs) and pregnant women were asked to agree or decline audio recording their smoking-related consultations. Data collected included percentage providing consent, number of recordings performed, HP type, and date (pre/post intervention). Interviews were conducted to assess the trial procedures' acceptability. Results. Two services provided seven recordings, all pre-intervention. Of the 22 recruited women, 14 consented to being audio recorded (64\%) and five provided recordings; of the 23 recruited HPs, 16 agreed (69\%), and two provided recordings. Qualitative data suggest that HPs found audio recording difficult to remember. HPs spent on average two minutes discussing smoking and used few BCTs. Conclusions. Audio recordings of smoking-related counselling were not feasible as planned. Future research will need to explore acceptable methods to assess BCT use in primary care.
\end{abstract}

\section{Introduction}

Tobacco smoking during pregnancy remains a global public health issue, specifically among disadvantaged populations such as minority groups, those with low socioeconomic status, and Indigenous populations [1]. Smoking rates among Australian Aboriginal and Torres Strait Islander pregnant women significantly declined in recent years, but are still threefold higher than the general population $(42 \%$ versus $11 \%$ in 2016) [2].

Behavioural counselling is an effective method to improve smoking cessation rates during pregnancy [3]. A meta-analysis of 30 studies found that counselling increased smoking cessation rates by $44 \%$ (RR $1.44,95 \%$ CI 1.19 to 1.73) [3]. Clinical guidelines recommend primary care health providers (HPs) provide brief behavioural counselling $[4,5]$. Guidelines sometimes use a broad term such as "assist with counselling" without further details. Most smoking cessation interventions do not provide a full description of what was included as part of counselling.

Recent novel research has tried to articulate the "active ingredients" of behavioural counselling, defined as behaviour change techniques (BCTs). A BCT is defined as an observable and replicable component designed to change behaviour [6], for example, "instruction on how to perform a behaviour." A BCT taxonomy has been developed and validated, which includes several categories of BCTs such as those that pertain to goals and planning, feedback and monitoring, social support, repetition and substitution, and regulation and more [6]. 
A specific taxonomy for smoking cessation has also been developed which includes forty-three possible BCTs [7]; several of them have been suggested as "evidence based" (defined as included in at least two interventions found to be effective in randomized controlled studies and/or associated with biochemically validated abstinence) [8, 9]. A few studies focused specifically on BCTs for smoking cessation in pregnancy, identifying 23 possibly effective BCTs [10, 11]. It is not yet clear which BCT combinations are effective in what context, setting, and subpopulations.

Understanding which BCTs are actually used during smoking cessation consultations can help guide future interventions. Research on smoking cessation behavioural interventions has focused on coding BCTs based on their description in published manuscripts or intervention manuals $[10,11]$. A major limitation is fidelity, as it is not always clear whether BCTs were performed as instructed, impacting also the ability to assess the mechanism through which an intervention is effective or not $[10,11]$. Identifying actual BCT use can provide objective data for this purpose. Few studies have recorded consultations to analyse which BCTs were employed; those that did were in specialized smoking cessation services $[8,12]$. Analysis of recorded consultations has not yet occurred in primary care settings, nor been used to identify effective BCTs for smoking cessation among Indigenous people, including pregnant women.

The aim of this study was to assess the feasibility of audio recording HP-pregnant women's smoking cessation behavioural counselling as part of an intervention in a primary care setting. A secondary aim was to conduct a preliminary exploration of the number and type of BCTs delivered as part of smoking cessation support to pregnant Aboriginal women.

\section{Materials and Methods}

2.1. Design. The study was a nested feasibility study within a larger intervention trial-the Indigenous Counselling and Nicotine (ICAN) Quit in Pregnancy [13-15]. The ICAN Quit in Pregnancy trial is aimed at testing the feasibility of an intervention to improve HP smoking cessation care with pregnant Aboriginal and Torres Strait Islander women who smoke in partnership with Aboriginal Medical Services [13-15]. The intervention included an educational resource package, webinar training, and free nicotine replacement therapy. Both the training and the educational resource package put an emphasis on specific BCTs which could support pregnant women to quit smoking, such as goal setting, problem solving, and boosting self-efficacy [13]. As part of the ICAN Quit in Pregnancy trial, both HPs and pregnant women were recruited and followed (November 2016 to September 2017). HPs were asked to complete a pre- and postintervention survey. Pregnant women were asked to complete surveys and undergo breath carbon monoxide measurements (for smoking status biochemical validation) at baseline and 4-week and 12-week follow-up visits. In each service, one health professional (who also saw pregnant women as part of their routine role) was nominated by the service as a research facilitator (and received additional training regarding research procedures, on top of the smoking cessation support training provided to all HPs). The research facilitator coordinated all of the ICAN Quit in Pregnancy trial components within the service, including recruiting patients, conducting the surveys and evaluations, and collecting the feasibility data. A full description of the ICAN Quit in Pregnancy trial methodology and results has been previously published, including adherence to the CONSORT guidelines [13-15].

2.2. Aboriginal Advisory Panel. The ICAN Quit in Pregnancy trial was developed collaboratively with two Aboriginal Medical Services [16]. A Stakeholder and Consumer Aboriginal Advisory Panel guided the development and implementation of this study to ensure Aboriginal community ownership and cultural sensitivity. At least one member from each Aboriginal Medical Service was invited to participate on this panel [16]. Full details can be found elsewhere [13, 14]. The Aboriginal Advisory Panel also guided the development of the nested study described here.

2.3. Setting. The study was conducted in six Aboriginal Medical Services-one urban and five regional-in three Australian states (New South Wales, Queensland, and South Australia).

2.4. Procedure. HPs and pregnant women recruited to the ICAN Quit in Pregnancy trial were asked, as part of the consent process, to agree or decline an additional option of audio recording the part of their consultations relating to smoking cessation. Defining the audio recording as an additional option was deemed necessary and more appropriate by the Stakeholder and Consumer Aboriginal Advisory Panel as it was felt that audio recordings might be perceived as more intrusive by Aboriginal women and might deter them from consenting to the ICAN Quit in Pregnancy trial. Taking into consideration that pregnant women discuss multiple pregnancy and nonpregnancy-related issues during their visits to the Aboriginal Medical Service, the Stakeholder and Consumer Aboriginal Advisory Panel found it to be unacceptable to record the entire session in order to extract only the smoking-related counselling. Therefore, the staff was instructed to record only the content regarding smoking during any visit of the recruited pregnant woman, if both HP and the woman had consented to recordings. This was done to encourage pregnant women's consent to the recording and protect privacy for other issues. All content was deidentified regarding the women and HPs. Research facilitators were provided with two audio recorders and were requested to coordinate with the other HP recording of a mix of initial and follow-up consultations (i.e., prequit attempt and postquit attempt up to the 4-week visit) with at least three pregnant women (expected recruitment for the ICAN Quit in Pregnancy trial was 10 eligible consenting women per service) [13], for a total of nine audio recordings per service.

2.5. Participants. HPs were eligible if they consulted with pregnant women either for pregnancy confirmation, antenatal care, and/or routine care. Pregnant women were eligible if they were $\leq 28$ weeks of gestation, Aboriginal and/or Torres Strait Islander or expectant mothers of Aboriginal and/or Torres Strait Islander babies, aged $\geq 16$ years old, and smoked 
tobacco at any level of consumption. Participating women received up to $\$ 60$ AUD as reimbursement for their time for ICAN Quit in Pregnancy trial-related visits. Women or HPs did not receive additional reimbursement for audiorecording consultations.

2.6. Qualitative Interviews. At the end of the ICAN Quit in Pregnancy trial, semistructured interviews were conducted with staff from the services (including managers, research facilitator, and HPs) to assess the acceptability of the study and intervention. No direct questions regarding feasibility and acceptability of audio-recording smoking cessationrelated consultations were included due to time constraints. However, a few general questions were included regarding what study procedures worked well and/or what were the more challenging aspects (including "How did the study go for you? What were the best things about the project? What went really well? What were some of the more challenging aspects? How did you manage these challenges? What impact did it have on your daily practice?").

\subsection{Analysis}

2.7.1. Feasibility Measures. These were collected by the research facilitator, including proportion consenting to audio recording, number of recordings performed, profession of HP (general practitioner/midwife/nurse/Aboriginal health worker) performing the consultation, and date (pre/post intervention).

2.7.2. Qualitative Interviews. Interviews at the end of the ICAN Quit in Pregnancy trial were audio recorded and transcribed. Data relevant to the feasibility and acceptability of audio-recording smoking cessation-related consultations was extracted.

2.7.3. Behaviour Change Techniques. Recordings were transcribed by a professional service and coded independently by two certified BCT coders (YBZ and ES). Patients' responses to the HP were not coded. Coding was based on Michie et al.'s 2010 taxonomy of smoking cessation BCTs $\mathrm{v} 1$, which includes the operational definition of each BCT, categorized under BCTs that address motivation, maximizing self-regulatory capacity/skills, promoting adjuvant activities, and general aspects of the interaction focusing on delivery of the intervention, information gathering, and communication $[7,10]$. Initial interrater agreement level (\% positive agreement) was $48 \%$ (calculated by identifying the proportion of all BCTs within a transcript that were recognized by both coders). Discrepancies were resolved through discussion, until agreement reached $100 \%$.

2.8. Ethics. The study was approved by the following ethics committees: (1) University of Newcastle Human Research Ethics Committee (HREC) (Reference H-2015-0438), (2) Aboriginal Health \& Medical Research Council HREC (Reference \#1140/15), (3) South Australia Aboriginal Health HREC (Reference \#04-16-652), and (4) Far North Queensland HREC (Reference \#16/QCH/34-1040). All participants provided and signed an informed consent to participate in the study.

\section{Results}

3.1. Feasibility Measures. In total, 22 pregnant women were recruited to the ICAN Quit in Pregnancy trial; 14 provided consent to audio recordings (64\%) (Figure 1). Of the 50 recruited HPs, we have data regarding agreement to audio recording for 23, with 16 providing consent $(69.5 \%$, 32\% from all HPs). Two Aboriginal Medical Services (of six) provided seven audio recordings, from two HPs and five women, all from the pre-intervention period. One service provided three recordings, with three pregnant women (one recording per woman), all with the same midwife. The second service provided four recordings, with two women (two recordings per woman), all with the tobacco action worker (a HP employed to assist with smoking prevention and cessation activities), who was also the research facilitator.

3.2. Qualitative Interviews. A total of eighteen interviews were conducted from all six Aboriginal Medical Services at the end of the ICAN Quit in Pregnancy trial (Figure 1). The topic of audio-recording consultations was discussed in two interviews, one with the research facilitator at a service that provided three audio recordings and another with the manager of a service that did not provide any audio recordings. Women's consent to the audio recordings varied considerably, with one service reporting having no issues "even signing video-voice recording consents, you know, they weren't finicky about it," and the other service stating none of the women found it acceptable "No, they didn't want it recorded." In the service that did provide audio recordings, these were still considered a difficult task to remember: "The doctors and midwives quite often forgot to use voice recorder. The GPs didn't remember very often that they had the voice recorders. Obviously very busy as well, so that, you know, it's understandable."

3.3. Behaviour Change Techniques. On average, the two HPs spent two minutes per consultation discussing smoking with the pregnant woman (range 00:47-03:47 minutes). They used few BCTs for each consultation (average 4 BCTs, range 2-8) (Table 1). The most common BCTs used were "building general rapport," "general communication approaches," and "information gathering and assessment." Supplementary Table S1 provides a summary of all of BCTs used, with example quotes.

In one consultation, the HP (tobacco action worker) was passive in response to the woman's enquiry, not taking advantage of her interest to further the discussion around smoking cessation.

Woman: "Hopefully I can quit once the baby is born. I mean I'd rather quit before the baby is born but...." HP: "Yeah well we see how it goes. You seem like you're giving it... something in here might help." Woman: "Oh is that the smoking program. Smoking cessation program?" HP: "Yes it is." Woman: "Isn't it kind of like this?" HP: "It's too early to be asking questions."

\section{Discussion}

4.1. Main Findings. In this nested study within six Aboriginal Medical Services, using audio recording of HP-pregnant 


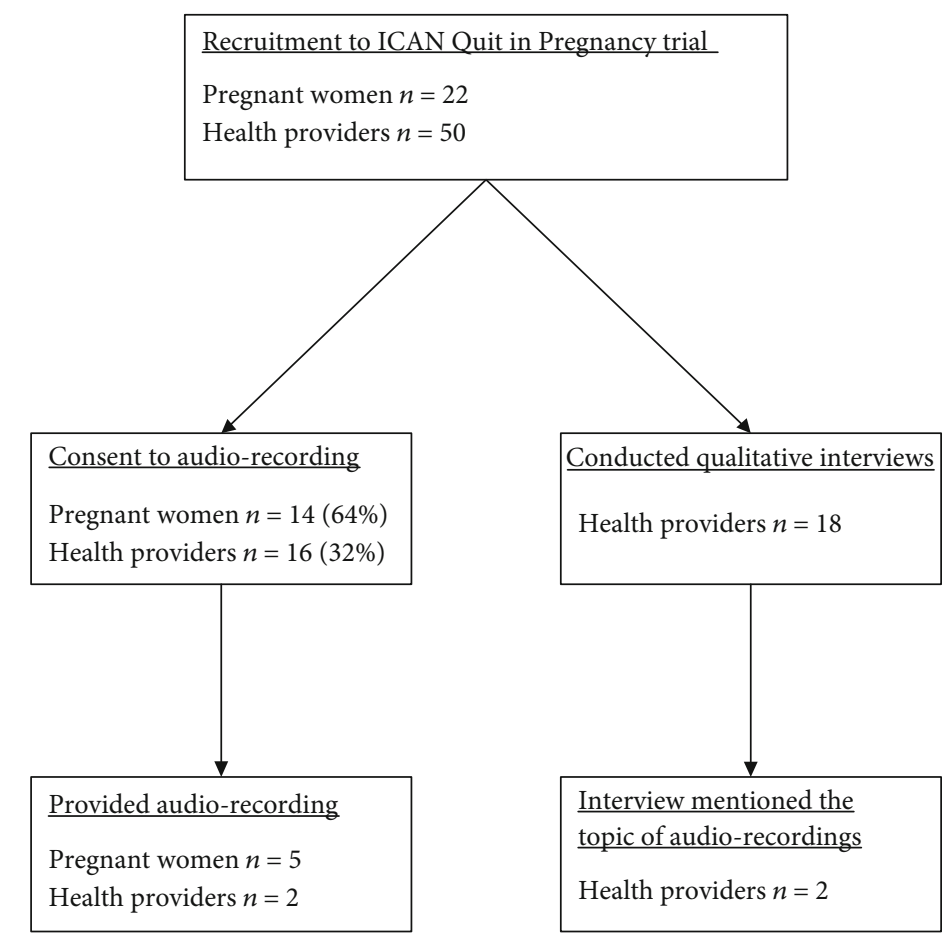

FIGURE 1: Flowchart of pregnant women and health providers' participation.

women's smoking counselling was not feasible as planned. Despite a reasonable rate of agreement to the consultations being recorded, very few recordings were obtained, and all of them in the pre-intervention trial phase (i.e., pre-training). Analysis of the few recordings that were provided showed that HPs devoted little time to smoking cessation support and used few evidence-based BCTs.

4.2. Comparison with the Literature. Audio recording of HPpatient visits as a way to assess actual provision of care and communication has been used successfully in the past, including in the context of smoking cessation counselling at specialist services $[8,12,17]$. Lorencatto et al. analysed 15 transcribed audio recordings from three smoking cessation services to develop the original methodology for specifying BCTs in practice [8]. Another study used 34 transcripts of audio recordings from two smoking cessation services to examine fidelity of treatment manuals and actual practice [12]. To the best of our knowledge, these recordings were of the entire patient visit and have not been used specifically with pregnant women or in an Indigenous context. In our study, the entire visit could not be recorded (following the advice of our Stakeholder and Consumer Aboriginal Advisory Panel). This may have reduced the practicality of obtaining audio recordings as HPs needed to stop the visit artificially and remember to start (and stop) recording when discussing smoking.

The initial interrater agreement level was low compared to that reported in other similar studies [8, 12, 17], despite the fact that both coders were trained and certified. This highlights the need for better training and using at least two coders for all transcripts with an iterative process in future studies.
The current Australian guidelines recommend that all HPs ask pregnant women regarding tobacco use, advise pregnant women who smoke to quit smoking and provide information on the most effective methods, and help them to quit by referring them to a Quitline, encouraging them to use behavioural strategies and using nicotine replacement therapy when appropriate [18]. Depending on the time available to the HP, guidelines recommend providing behavioural counselling which can include, among other things, strategies for coping with smoking triggers, addressing barriers to quitting, and setting a quit date [18]. Currently, smoking cessation support during pregnancy is suboptimal [19-21], with previous research suggesting that HPs might be concerned that raising the issue will damage their therapeutic relationship; therefore, HPs put an emphasis on building a positive rapport with the women, being nonjudgmental and supportive [20-22]. The findings from this study provide further support for this as "building general rapport" was one of the most common BCTs used. BCTs that were found in previous research $[10,11,23]$ to be associated with cessation success such as "facilitate barrier identification and problem solving," "facilitate action planning/identify relapse trigger," and "facilitate goal setting" were not used. Future interventions with HPs should focus on skills training of "how" to assist pregnant women to quit using other BCTs that have been suggested as effective in pregnancy [11, 23, 24], while being nonjudgmental and supportive. The ICAN Quit in Pregnancy training laid emphasis on these skills; unfortunately, no recordings were taken to inform whether the training had been successful or HP fidelity with the approach.

Using more BCTs has been previously suggested to yield better results [23]. Time is perceived as a leading barrier to providing adequate smoking cessation support in primary 
TABLe 1: Behaviour change techniques used in audio-recorded consultations as part of ICAN Quit in Pregnancy trial.

\begin{tabular}{|c|c|}
\hline Consultation number and time recorded (minutes) & Behaviour change techniques (BCTs) used \\
\hline \multicolumn{2}{|l|}{ Service \#1: consultations with midwife } \\
\hline $\begin{array}{l}\text { Patient \#1 } \\
(02: 05)\end{array}$ & $\begin{array}{l}\text { Six BCTs: } \\
\text { (i) RC1: build general rapport } \\
\text { (ii) RC2: general practitioner communication approaches } \\
\text { (iii) RC6: offer/direct towards appropriate written materials } \\
\text { (iv) BM3: provide feedback on current behaviour and progress } \\
\text { (v) BM11: measure CO and explain the purposes of CO monitoring } \\
\text { (vi) A1: advise on stop smoking medication }\end{array}$ \\
\hline $\begin{array}{l}\text { Patient \#2 } \\
(00: 48)\end{array}$ & $\begin{array}{l}\text { Three BCTs: } \\
\text { (i) RC7: information gathering and assessment } \\
\text { (ii) BM5: provide normative information about others' } \\
\text { behaviour and experiences } \\
\text { (iii) BM9: facilitate identification of reasons for wanting } \\
\text { and not wanting to stop smoking }\end{array}$ \\
\hline $\begin{array}{l}\text { Patient \#3 } \\
(03: 47)\end{array}$ & $\begin{array}{l}\text { Four BCTs: } \\
\text { (i) RC7: information gathering and assessment } \\
\text { (ii) A1: advise on stop smoking medication } \\
\text { (iii) A3: ask about experiences of stop smoking medication } \\
\text { that the smoker is currently using } \\
\text { (iv) BS11: advise on avoiding social cues for smoking }\end{array}$ \\
\hline
\end{tabular}

Service \#2: consultations with tobacco action worker

\begin{tabular}{ll} 
& Five BCTs: \\
Patient $\# 4$ & (i) RC1: build general rapport \\
First visit & (ii) RC2: general practitioner communication approaches \\
$(02: 55)$ & (iii) RC7: information gathering and assessment \\
& (iv) BM3: provide feedback on current behaviour and progress \\
& (v) BM7: provide rewards contingent on effort or progress \\
Patient $\# 4$ & Two BCTs: \\
Second visit & (i) RC1: build general rapport \\
$(00: 47)$ & (ii) BM13: create or reinforce negative associations \\
Patient $\# 5$ & Three BCTs: \\
First visit & (i) RC1: build general rapport \\
$(01: 13)$ & (ii) RC7: information gathering and assessment \\
Patient $\# 5$ & (iii) BM7: provide rewards contingent on effort or progress \\
Second visit & Two BCTs: \\
$(02: 25)$ & (i) RC1: build general rapport \\
\hline & (ii) RC7: information gathering and assessment
\end{tabular}

care $[22,23]$. In this study, HPs spent on average only two minutes on their smoking-related consultation. Therefore, it is essential to understand which combination of effective BCTs would be feasible in this busy setting.

4.3. Strengths and Limitations. This is the first study that explored smoking cessation-related BCTs used in primary care settings with pregnant Aboriginal and Torres Strait Islander women. It is not possible to generalize the findings from the few audio recordings that were collected. There may have been longer consultations around smoking that included other important issues impacting smoking that women did not want recorded. Other HPs may have used other BCTs or more BCTs. Furthermore, as only pretraining audio recordings were collected, it was not possible to explore whether the intervention had any impact on the length or BCT content of the consultations. Data regarding the reasons why women and/or HPs did not consent to the audio recording, or did not perform the audio recording (for example, whether another person accompanying the pregnant woman was present during the consultation), were not collected, impacting the ability to fully understand the factors influencing the feasibility and acceptability of the audio recording.

4.4. Implications for Future Research and Practice. The results of this study will be used to inform a larger cluster randomized controlled study (SISTAQUIT ${ }^{\circledR}$ ), in up to 30 Aboriginal Medical Services, which is also attempting to collect audio recordings of smoking-related counselling and compare BCT use both pre- and post-intervention, between the control and intervention groups. Comparing BCT use between different HPs, such as GPs, midwifes, and tobacco action workers, is also important and needs to be taken into 
consideration when designing future research. Suggested changes to increase the feasibility of obtaining recordings could include the following:

(1) Implementing a computerized system to flag participating woman that agreed to be audio recorded, including a reminder at the beginning of the visit

(2) Supplying each service with more audio recorders (one for each HP)

(3) Providing an incentive for both HP and the women to perform the recording

(4) Exploring other ways that might be more feasible and acceptable to the communities such as using observation (without audio recording) by training the research facilitator in $\mathrm{BCT}$ identification

(5) Using HP self-report of BCTs with a template

\section{Conclusions}

Recording HP consultations with pregnant women about their smoking was not feasible as planned. Analysis of the few recordings that were provided showed that HPs devoted little time to smoking cessation and used few evidence-based BCTs. Future research will need to explore feasible ways to assess BCT use in primary care and specifically in Aboriginal health services, including which BCT combination would be the most effective in time-deprived primary care settings.

\section{Data Availability}

No data is available.

\section{Ethical Approval}

The authors assert that all procedures contributing to this work comply with the ethical standards of the relevant national and institutional committees on human experimentation and with the Helsinki Declaration of 1975, as revised in 2008 .

\section{Disclosure}

Funding agencies did not have any role in the design of the study; collection, analysis, and interpretation of data; and in writing the manuscript.

\section{Conflicts of Interest}

YBZ has received fees for lectures from Pfizer Ltd. Israel (2012-2015, 2017-2019) and GSK Consumer Health Israel (2019) (both distributing smoking cessation pharmacotherapies in Israel). She has not received any fees from pharmaceutical companies in Australia. No other coauthors have conflicts of interest.

\section{Acknowledgments}

The authors thank the Aboriginal communities who have contributed to the development and implementation of the ICAN Quit in Pregnancy intervention. This includes staff and patients of Biripi Aboriginal Corporation, Tobwabba Aboriginal Medical Service, Pangula Mannamurna Aboriginal Corporation, Riverina Medical and Dental Aboriginal Corporation, Wuchopperen Health Service Ltd., and Tharawal Aboriginal Corporation. The authors thank the members of the Stakeholder and Consumer Aboriginal Advisory Panel for ICAN Quit in Pregnancy for the ongoing guidance and support in implementing the study. We would also like to acknowledge Lauren Pollack who worked as a research assistant throughout the study and was responsible for the everyday administration of running this study. This work was supported by the NSW Ministry of Health and the Hunter Cancer Research Alliance. YBZ was supported by the University of Newcastle and Hunter Cancer Research Alliance PhD scholarship. MB was supported by the University of Newcastle and Australian Heart Foundation Indigenous Scholarship (\#101555). GG is supported by National Health and Medical Research Council and Cancer Institute NSW Early Career Research Fellowships (APP1092085 and 15ECF/I-52).

\section{Supplementary Materials}

Table S1: a summary of all of the behaviour change techniques (BCTs) used with examples of quotes. (Supplementary Materials)

\section{References}

[1] S. Lange, C. Probst, J. Rehm, and S. Popova, "National, regional, and global prevalence of smoking during pregnancy in the general population: a systematic review and meta-analysis," The Lancet Global Health, vol. 6, no. 7, pp. e769-e776, 2018.

[2] Australian Institute of Health and Welfare, Australia's Mothers and Babies 2016-In Brief, Australian Institute of Health and Welfare, Canberra, 2018.

[3] C. Chamberlain, A. O'Mara-Eves, J. Porter et al., "Psychosocial interventions for supporting women to stop smoking in pregnancy," Cochrane database of systematic reviews, vol. 2, article CD001055, 2017.

[4] N. Zwar, R. Richmond, R. Borland et al., Supporting Smoking Cessation: A Guide for Health Professionals. Melbourne: The Royal Australian College of General Practitioners, 2011.

[5] The American College of Obstetricians and Gynecologists, "Committee Opinion No 721: smoking cessation during pregnancy," Obstetrics and Gynecology, vol. 130, no. 4, pp. e200e204, 2017.

[6] S. Michie, C. E. Wood, M. Johnston, C. Abraham, J. J. Francis, and W. Hardeman, "Behaviour change techniques: the development and evaluation of a taxonomic method for reporting and describing behaviour change interventions (a suite of five studies involving consensus methods, randomised controlled trials and analysis of qualitative data)," Health Technology Assessment, vol. 19, no. 99, pp. 1-188, 2015.

[7] S. Michie, N. Hyder, A. Walia, and R. West, "Development of a taxonomy of behaviour change techniques used in individual 
behavioural support for smoking cessation," Addictive Behaviors, vol. 36, no. 4, pp. 315-319, 2011.

[8] F. Lorencatto, R. West, N. Seymour, and S. Michie, "Developing a method for specifying the components of behavior change interventions in practice: the example of smoking cessation," Journal of Consulting and Clinical Psychology, vol. 81, no. 3, pp. 528-544, 2013.

[9] R. West, A. Evans, and S. Michie, "Behavior change techniques used in group-based behavioral support by the English stopsmoking services and preliminary assessment of association with short-term quit outcomes," Nicotine \& Tobacco Research, vol. 13, no. 12, pp. 1316-1320, 2011.

[10] F. Lorencatto, R. West, and S. Michie, "Specifying evidencebased behavior change techniques to aid smoking cessation in pregnancy," Nicotine \& Tobacco Research, vol. 14, no. 9, pp. 1019-1026, 2012.

[11] K. A. Campbell, L. Fergie, T. Coleman-Haynes et al., "Improving behavioral support for smoking cessation in pregnancy: what are the barriers to stopping and which behavior change techniques can influence these? Application of Theoretical Domains Framework," International journal of environmental research and public health, vol. 15, no. 2, 2018.

[12] F. Lorencatto, R. West, C. Christopherson, and S. Michie, "Assessing fidelity of delivery of smoking cessation behavioural support in practice," Implementation Science, vol. 8, article 40, 2013.

[13] Y. Bar-Zeev, B. Bonevski, M. Bovill et al., "The Indigenous Counselling and Nicotine (ICAN) QUIT in Pregnancy Pilot Study protocol: a feasibility step-wedge cluster randomised trial to improve health providers' management of smoking during pregnancy," BMJ Open, vol. 7, no. 8, 2017.

[14] G. S. Gould, M. Bovill, L. Pollock et al., "Feasibility and acceptability of Indigenous Counselling and Nicotine (ICAN) QUIT in pregnancy multicomponent implementation intervention and study design for Australian Indigenous pregnant women: a pilot cluster randomised step-wedge trial," Addictive Behaviors, vol. 90, pp. 176-190, 2019.

[15] Y. Bar-Zeev, M. Bovill, B. Bonevski et al., "Improving smoking cessation care in pregnancy at Aboriginal Medical Services: 'ICAN QUIT in Pregnancy' step-wedge cluster randomised study," BMJ Open, vol. 9, no. 6, article e025293, 2019.

[16] M. Bovill, Y. Bar-Zeev, M. Gruppetta, P. O'Mara, B. Cowling, and G. S. Gould, "Collective and negotiated design for a clinical trial addressing smoking cessation supports for Aboriginal and Torres Strait Islander mothers in NSW, SA and Qlddeveloping a pilot study," Australian Journal of Primary Health, vol. 23, pp. 497-503, 2017.

[17] P. J. Lawson, S. A. Flocke, and B. Casucci, "Development of an instrument to document the 5A's for smoking cessation," American Journal of Preventive Medicine, vol. 37, no. 3, pp. 248-254, 2009.

[18] Royal Australian College of General Practitioners, Supporting Smoking Cessation: A Guide for Health Professionals, RACGP, East Melbourne, Vic, 2nd ed edition, 2019.

[19] Y. B. Zeev, B. Bonevski, L. Twyman et al., "Opportunities missed: a cross-sectional survey of the provision of smoking cessation care to pregnant women by Australian general practitioners and obstetricians," Nicotine \& Tobacco Research, vol. 19, no. 5, pp. 636-641, 2017.

[20] M. E. Passey, C. A. D'Este, and R. W. Sanson-Fisher, "Knowledge, attitudes and other factors associated with assessment of tobacco smoking among pregnant Aboriginal women by health care providers: a cross-sectional survey," BMC Public Health, vol. 12, article 165, 2012.

[21] J. M. Longman, C. M. Adams, J. J. Johnston, and M. E. Passey, "Improving implementation of the smoking cessation guidelines with pregnant women: how to support clinicians?," Midwifery, vol. 58, pp. 137-144, 2018.

[22] Y. Bar-Zeev, E. Skelton, B. Bonevski, M. Gruppetta, and G. S. Gould, "Overcoming challenges to treating tobacco use during pregnancy - a qualitative study of Australian general practitioners barriers," BMC Pregnancy and Childbirth, vol. 19, no. 1, p. 61, 2019.

[23] S. E. Griffiths, J. Parsons, F. Naughton, E. A. Fulton, I. Tombor, and K. E. Brown, "Are digital interventions for smoking cessation in pregnancy effective? A systematic review and metaanalysis," Health Psychology Review, vol. 12, no. 4, pp. 333356, 2018.

[24] L. Fergie, K. A. Campbell, T. Coleman-Haynes, M. Ussher, S. Cooper, and T. Coleman, "Stop smoking practitioner consensus on barriers and facilitators to smoking cessation in pregnancy and how to address these: a modified Delphi survey," Addictive Behaviors Reports, vol. 9, article 100164, 2019. 